\title{
The Effects of Lentivirus-mediated shRNA Interference Targeting Mcl-1 on Growth of NK/T-cell Lymphoma
}

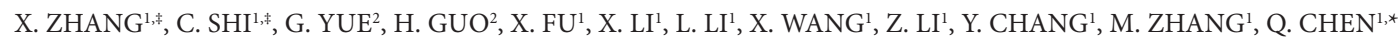 \\ ${ }^{1}$ Department of oncology, The First Affiliated Hospital of Zhengzhou University, No.1 Jianshe East Road, Zhengzhou Henan 450052, \\ China; ${ }^{2}$ Department of oncology, The Affiliated Cancer Hospital of Zhengzhou University, No. 127 Dong Ming Road, Zhengzhou Henan \\ 450008, China \\ ${ }^{*}$ Correspondence: qingjiang_c@126.com. \\ ${ }^{*}$ Contributed equally to this work.
}

Received November 8, 2016 / Accepted January 7, 2017

\begin{abstract}
Myeloid leukemia-1 (Mcl-1) gene has been reported as an important factor in various types of cancer, but little research was processed on natural killer (NK)/T-cell lymphoma, a kind of a highly aggressive disease with a poor prognosis. Here we investigated the expression of Mcl-1 in seven lymphoma cell lines and its potential role as a molecular drug target for NK/T-cell lymphoma therapy by using lentivirus-mediated shRNA interference targeting Mcl-1 (lenti-shMcl-1). In our study, the expression of Mcl-1 in different lymphoma cell lines were evaluated firstly, after that lenti-shMcl-1 was constructed and transduced into NK/T-cell lymphoma cell line SNK-6 which had a high level expression of Mcl-1. Methylthiazolete-Trazolium (MTT) assay and flow cytometry (FCA) were employed to detect the status of proliferation and apoptosis after infection. Lastly we investigated the effects of chemotherapy agent vincristine (VCR) combination with lenti-shMcl-1 by MTT and FCA assay. The results showed that Mcl-1 gene expressed in all seven lymphoma cell lines at different levels. Recombinant lentiviruses could infect SNK-6 cells effectively. Mcl-1 expression level was remarkably down-regulated after infection with lenti-shMcl-1. The growth of SNK-6 cells was inhibited significantly through apoptosis pathway. Otherwise, lenti-shMcl-1 also revealed a significant chemosensitizing effect in combination with vincristine. In a word, we demonstrated that lentishMcl-1 had a significant anti-NK/T cell lymphoma effect and targeting Mcl-1 therapy could be a promising novel approach in treatment of lymphoma.
\end{abstract}

Key words: lymphoma, Mcl-1, shRNA, lentivirus, apoptosis

Lymphoma is the most common hematologic malignancy and comprises a heterogeneous group of malignancies consisting of different clinic pathologic entities [1,2]. NK/T-cell lymphoma is a distinct subtype of lymphoma relatively common in Asia and South America. Histological features of this disease are angiocentricity, angiodestruction, and massive necrosis and apoptosis. Although most cases initially present with localized disease, NK/T-cell lymphoma is highly aggressive and resistant to chemotherapy with a poor prognosis $[3,4]$. Moreover, many traditional treatments usually lead to a lot of toxicities which could affect the therapeutic outcome and quality of life [5]. Thus, seeking a new strategy for the treatment of NK/T-cell lymphoma is needed.

In the last few years a number of promising new therapies including gene therapy have been developed. RNA interference
(RNAi) by viral vector is a new form of gene therapy. There are many advantages about viral vectors for RNAi compared to non-viral vectors, for example, high transfection efficiency, broad host range and long-term expression of therapeutic gene [6]. ShRNA expression viral vectors such as retroviral vectors, adenoviral vectors and, more recently, lentiviral vectors had been reported and proven to be safe and effective for humans with no obvious side effects in many solid tumors [7]. It had been reported that lymphoma cells had a low infection rate with most viral vectors. Thus finding an appropriate viral vector for lymphoma cells is the first step in gene therapy. Our previous researches had demonstrated that lentivirus could be an ideal vector for transducing heterologous gene into lymphoma cells $[8,9]$. So in this study we employed lentivirus which contains pLL3.7 plasmid as our vector. PLL3.7 has a mU6 promoter that could express shRNA efficiently and 
CMV-EGFP component as report gene which can evaluate the efficiency of infection.

Mcl-1 is a pro-survival member of Bcl-2 family which could promote oncogenesis by inhibition of apoptosis [10]. Many kinds of cancer cells have been shown to be reliant on Mcl-1 for their survival and development. Substantial evidence showed that $\mathrm{Mcl}-1$ overexpression promoted oncogenesis recently, it had been reported in several haematological cancers and solid tumours, including leukemia [11] and hepatocellular carcinomas [12]. Forced overexpression of Mcl-1 in transgenic mice led to a significantly high incidence of B-cell lymphoma [13], while forced Mcl-1 down-regulation was able to induce apoptosis in a number of cancer cell types [14]. Overexpression of Mcl-1 also could lead to chemoresistance of certain cancers [15-17]. Thus Mcl-1 is an attractive and potential therapeutic target in a number of malignancies, and is the focus of a number of studies [18].

In our study we investigated the expression of Mcl-1 in seven lymphoma cell lines. Lentivirus-mediated shRNA targeting Mcl-1 which could effectively infect SNK-6 cells was successfully constructed. After recombinant lentivirus infection and combination with vincristine we explored its potential role as a molecular drug target for NK/T-cell lymphoma therapy.

\section{Materials and methods}

Lymphoma cell lines and culture. Human follicular B cell line DOHH2 and human NK lymphoma cell line YTS were kindly provided by Drs Scott Kaufmann and Daniel Billadeau, respectively, from Mayo Clinic, Rochester, Minnesota, USA. Human NK/T lymphoma cell line SNK-6 was granted by Professor Norio Shimizu and Yu Zhang of Chiba University. Human cutaneous T lymphoma cell line Hut-78, human lymphoblastic lymphoma cell line Jurkat and human Burkitt's lymphoma cell line Raji were obtained from Committee on Type Culture Collection of Chinese Academy of Sciences (Shanghai, China). Human anaplastic large cell lymphoma cell line Karpas 299 was obtained from Shanghai DoBio Biotech Company (Shanghai, China). SNK-6 cells were maintained in RPMI 1640 containing 10\% human AB serum and $700 \mathrm{U} / \mathrm{ml}$ recombinant interleukin-2. DOHH2, Hut-78, Jurkat, Karpas 299, Raji and YTS cells were maintained in RPMI 1640 containing 10\% fetal bovine serum. YTS cells were maintained in media containing $0.1 \mathrm{mM}$ nonessential amino acid.

Cell line for virus manipulation. Human embryonic kidney $293 \mathrm{~T}$ cell line was obtained from Committee on Type Culture Collection of Chinese Academy of Sciences and maintained in DMEM supplemented with 10\% fetal bovine serum and $0.1 \mathrm{mM}$ nonessential amino acid. All the cell lines were plated in a fully humidified atmosphere containing $5 \%$ $\mathrm{CO} 2$ at $37^{\circ} \mathrm{C}$. Cells in exponential phase of growth were used in our study.

RT-PCR analysis. Seven lymphoma cell lines were examined by RT-PCR for Mcl-1 expression. Total RNA from seven lymphoma lines was prepared with Trizol reagent. cDNA was synthesized with random primers following the manufacturer's protocol. Then Mcl-1 was amplified by PCR amplification kit. Two sets of primers were used as follows. Actin-F: 5'-CTG GGA CGA CAT AGG AGA AAA-3', ActinR: 5'-AAG GAA GGC TGG AAG AGT GC-3', with a product length of 564bp. Mcl-1-F: 5'-GTA AGG ACA AAA CGG GAC-3', Mcl-1-R: 5'-CTC TTC AAT CAA TGG GGA-3', with a product length of $350 \mathrm{bp}$. The results were analyzed by $\mathrm{Gel}$ electrophoresis image.

Construction and production of lentivirus. Lentivirus system consists of three packing vectors: pLL3.7, pCMV- $\Delta 8.2$ and pCMV-VSVG. Plasmid pLL3.7 contains a GFP reporter and a U6 promoter upstream of the cloning restriction sites (Hpa I and Xho I). SiRNA targeting Mcl-1(GCA GTC CTC TAG TGT TTC A) [19] and scrambled control were designed and synthesized. Then oligonucleotides were annealed, digested and inserted between Hpa I and Xho I restriction sites of pll3.7 plasmid named pll-shMcl-1 and pll-con. Correct insertions of shRNA cassettes were confirmed by restriction mapping and direct DNA sequencing. Pll-shMcl-1 and pll-con plasmids were then co-transfected with the other two plasmids into $293 \mathrm{~T}$ cells to generate individual lentiviruses. After $48 \mathrm{~h}$, lenti-shMcl-1 and lenti-con viruses were harvested and purified. Viral titer was determined by hole-by-dilution titer assay through infecting 293T cells [20].

Cells infection and assessment of efficiency. In our study, we chose SNK-6 as our target cells. For lentivirus infection, SNK-6 cells were cultured in 6-well plates with the concentration of $1 \times 10^{6} / \mathrm{ml}$ and then treated with lentivirus at multiplicity of infection (MOI) of 50. Cells were washed with PBS to remove unattached viruses after $3 \mathrm{~h}$ infection. Normal medium was added for further experiment. GFP expression was determined $96 \mathrm{~h}$ after infection by fluorescence microscopy.

Quantitative real-time PCR. We extract total RNA from the parental SNK-6 cells and SNK-6 cells $96 \mathrm{~h}$ after infection with lenti-shMcl-1 and lenti-con. cDNA was then synthesized. In quantitative real-time PCR, the primers were used as follows. Actin-F: 5'- AGC GAG CAT CCC CCA AAG TT -3', Actin-R: 5'- GGG CAC GAA GGC TCA TCA TT -3'; the primers of Mcl-1 are as described above. Real-time PCR was performed using a standard SYBR Green PCR kit protocol on Applied Biosystems 7500 Fast Real-Time System.

Western blotting analysis. We extract total protein from the parental SNK- 6 cells and SNK-6 cells $96 \mathrm{~h}$ after infection with lenti-shMcl-1 and lenti-con. The concentration of total protein was determined by BCA protein assay. Cell extracts from all samples were separated on $10 \%$ SDS-polyacrylamide gels and transferred electrophoretically to a polyvinylidene fluoride membrane, which were then blocked with 5\% skim milk. Membranes were incubated with specific primary antibodies for Mcl-1 or GAPDH, followed by incubation in secondary antibody and visualized by enhanced chemiluminescence. 
MTT assay. Cell proliferation was determined by methylthiazolete-trazolium (MTT) assay. SNK-6 cells were infected with lenti-shMcl-1 or lenti-con in 6-well plates and after $3 \mathrm{~h}$ infection they were reseeded in 96-well plates at an optimal density $\left(5 \times 10^{3}\right.$ cells per well). After 1, 2, 3 and 4 days, cells were stained with $20 \mathrm{~mL}$ MTT $(5 \mathrm{mg} / \mathrm{mL})$ and subsequently made soluble in $100 \mathrm{~mL}$ of DMSO. Absorbance was measured at $493 \mathrm{~nm}$ using a microtiter plate reader.

Flow cytometry assay. We seeded $1 \times 10^{6}$ cells in $6 \mathrm{~cm}$ dishes and infected with lenti-shMcl-1 and lenti-con for 1, 2,3 , and 4 days, then cells were collected, washed with PBS, and fixed with $70 \%$ cold ethanol. The fixed cells were treated with $50 \mu \mathrm{g} / \mathrm{ml}$ of DNase-free RNase and $20 \mu \mathrm{g} / \mathrm{ml}$ of propidiumiodide. A total of 10,000 cells were analyzed by FACScan. Hypochromatic cells due to chromatin condensation with sub-diploid DNA profiles were identified as apoptotic fraction of cells. Cell debris was excluded from analysis by appropriate light scatter gating.

Combination with vincristine. In pre-experiments we determined $0.01 \mu \mathrm{g} / \mathrm{ml}$ vincristine as a concentration resulting in an about $20 \%$ reduction of cell numbers of SNK-6 cells. SNK-6 cells were infected with recombinant lentivirus in 6-well plates for $96 \mathrm{~h}$ and then reseeded in 96-well plates at an optimal density $\left(1 \times 10^{4}\right.$ cells per well $)$, the cells were co-incubated with vincristine at the concentration of $0.01 \mu \mathrm{g} /$ $\mathrm{ml}$ for 1,2, 3 and 4 days. The effects of combination with vincristine were detected by MTT and FCA assay.

Statistical analysis. All of the data is expressed as mean \pm standard deviation of three experiments. Statistical significance of differences was determined by Student's $t$-test. A $P$ value of less than 0.05 was considered significant. These analyses were performed using SPSS 17.0 software.

\section{Results}

Expression of Mcl-1 in different kinds of lymphoma cell lines. The expression of Mcl-1 in seven different lymphoma cell lines was examined by RT-PCR assay and western blot. It revealed that Mcl-1 expressed widely in lymphoma cells. And the expression levels of Mcl-1 in these cell lines were different, SNK-6 and Karpas 299 expressed relatively higher than other cell lines $(P<0.05$, data was not shown. Figure 1$)$. In our study we chose SNK-6 as our target cells.

Construction of recombinant lentiviruses and infection of SNK-6 cells. ShRNA targeting Mcl-1 and scrambled control sequence were separately inserted into plasmid pll3.7 called pll-shMcl-1 and pll-con which were proved correct by direct DNA sequencing (Figure 2). Then pll-shMcl-1 and pll-con plasmids were co-transfected with the other two plasmids into $293 \mathrm{~T}$ cells to generate lentiviruses. After $48 \mathrm{~h}$, the recombinant lentiviruses lenti-shMcl-1 and lenti-con were harvested and purified. Results showed that the titer of recombinant lentiviruses reached to $10^{9} \mathrm{TU} / \mathrm{ml}$ without significant difference $(P>0.05)$.

We infected SNK-6 cells with lentivirus, lenti-shMcl-1 and lenti-con at MOI of 50. The expression of GFP was as an indicator for infection efficiency by microscope. As shown in Figure 2, over 70\% cells were GFP-positive for lenti-shMcl-1 group, indicating that a large proportion of cells were successfully infected. There are no significant differences in three groups $(P>0.05)$.

Expression of Mcl-1 after infection. We infected SNK-6 cells with lenti-shMcl-1 and lenti-con at MOI of 50. After 96h infection the knockdown efficiency of Mcl-1 was studied by real-time PCR $\left(2^{-\mathrm{DDCT}}\right)$ and Western blotting assay. In lentishMcl-1 group, the mRNA levels were decreased by $66.8 \%$ (Figure 3A). Also, the protein levels were remarkably decreased by lenti-shMcl-1, compared to the levels of housekeeping gene GAPDH (Figure 3B).

Effects of lenti-shMcl-1 on proliferation and apoptosis. In order to explore the effects of Mcl-1 on proliferation and apoptosis of NK/T lymphoma cells, MTT cell proliferation assay was performed for four successive days in three groups.

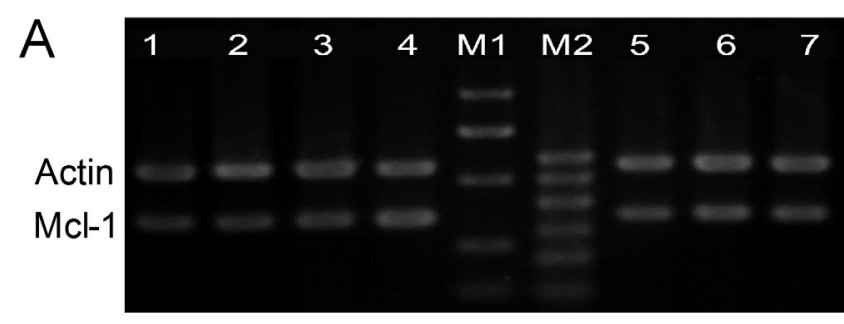

$B$
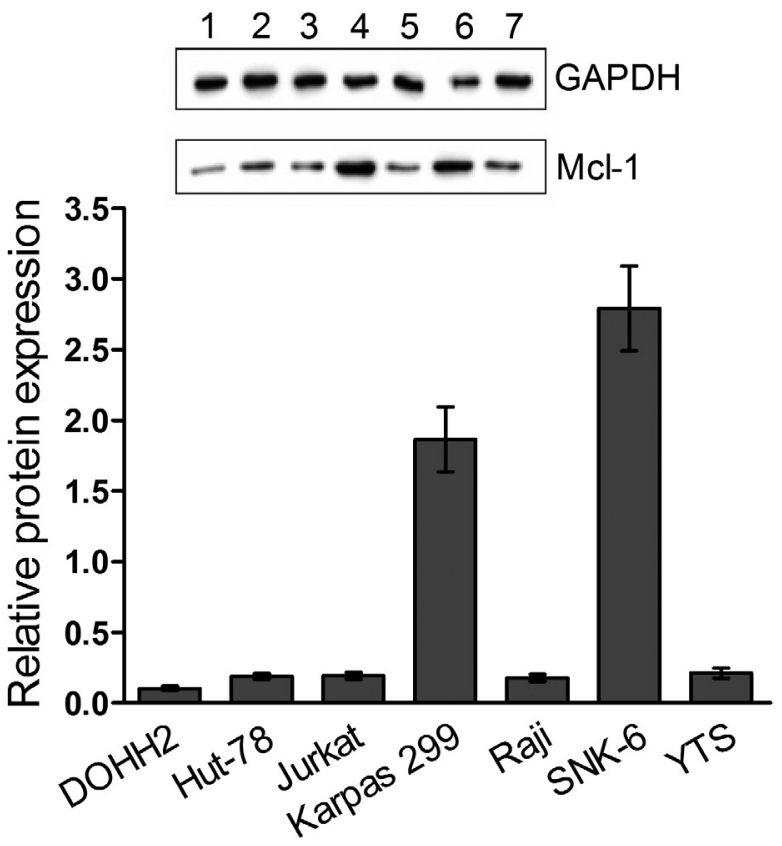

Figure 1. Mcl-1 mRNA (A) and protein (B) expression in human lymphoma line cells (1: DOHH2, 2: Hut-78, 3: Jurkat, 4: Karpas 299, 5: Raji, 6: SNK-6, 7: YTS). It revealed that Mcl-1 expressed in all seven lymphoma cell lines. SNK-6 and Karpas 299 expressed relatively higher than other cell lines $(P<\mathbf{0 . 0 5})$. 


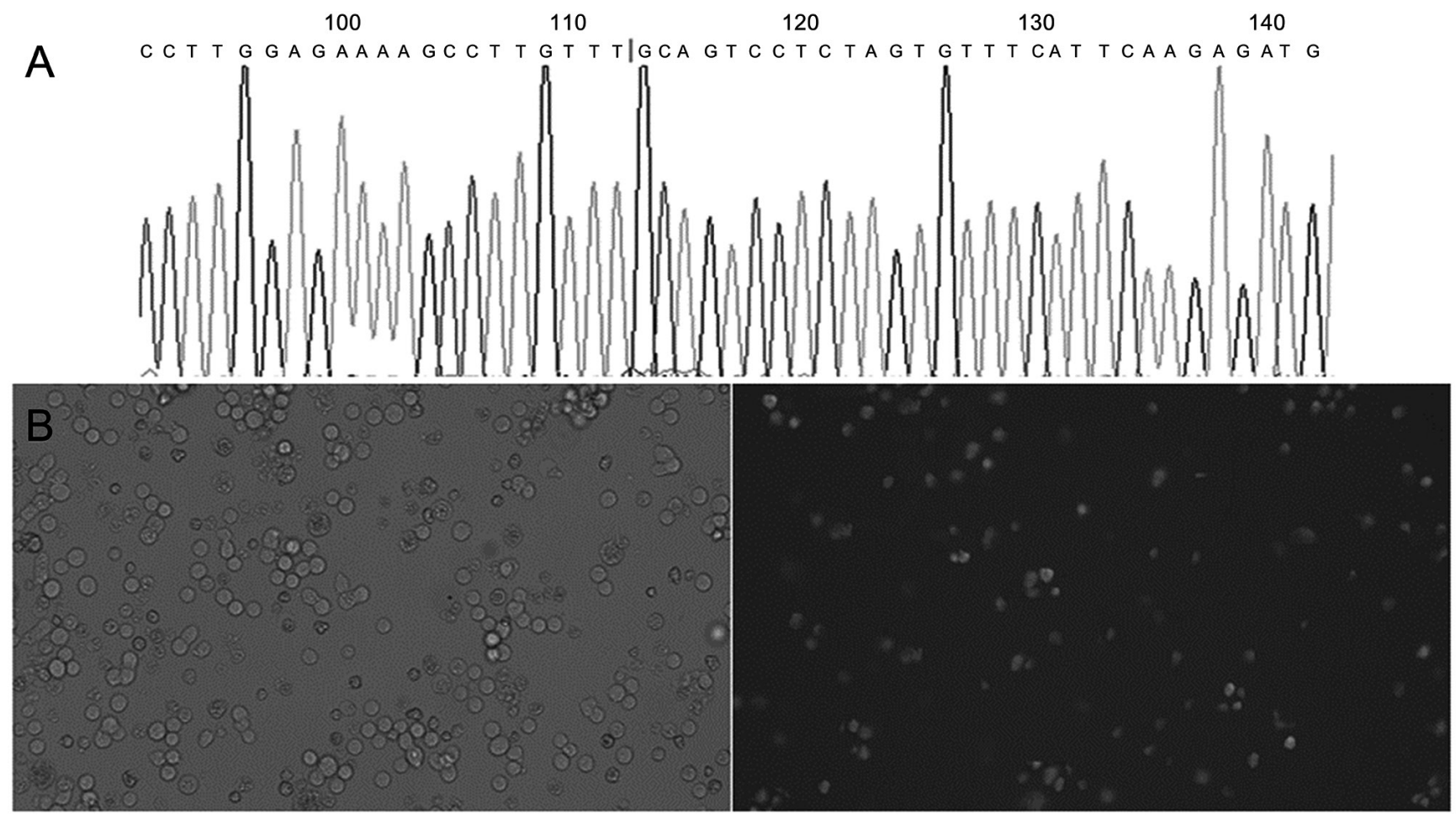

Figure 2. Construction of recombinant lentiviruses and infection of SNK-6 cells. (A) ShRNA targeting Mcl-1 was inserted into plasmid pll3.7 called pll-shMcl-1. Then it was proved correct by direct DNA sequencing. (B) Photomicrographs of GFP-positive SNK-6 cells infected by lenti-shMcl-1. Bright light and fluorescence pictures were taken $96 \mathrm{~h}$ after infection at MOI of 50; over $70 \%$ cells were GFP-positive for lenti-shMcl-1 group, indicating that a large proportion of cells were successfully infected.

The cell viability of lenti-shMcl-1 group decreased by $29.0 \%$ after 4 days infection compared to lenti-con group (Figure $4 \mathrm{~A}$ ). To further investigate the potential mechanism regulating the inhibition of growth by lenti-shMcl-1, the apoptosis status was determined by flow cytometry analysis. When Mcl-1 expression was down regulated the apoptosis rate of Mcl-1-shRNA infected cells were significantly higher than the lenti-con group $(26.7 \% \pm 2.7 \%$ vs $5.6 \% \pm 3.0 \%, P<0.05$, Figure $4 \mathrm{~B})$.

Combination of lenti-shMcl-1 and vincristine. SNK-6 cells were infected with recombinant lentivirus for $96 \mathrm{~h}$ and reseeded in 96-well plates at an optimal density, then the cells were co-incubated with vincristine at concentration of $0.01 \mu \mathrm{g} / \mathrm{ml}$ for $1,2,3$ and 4 days. The effects of three groups were detected by MTT and FCA assay. MTT assay suggested that the cell viability of SNK-6 cells was inhibited significantly by combination group against VCR single agent and VCR combined with lenti-con groups after 4 days $(35.7 \% \pm 2.5 \%$ vs $77.6 \% \pm 1.7 \%$ vs $75.0 \% \pm 1.8 \%, P<0.05$, Figure $5 \mathrm{~A})$. Flow cytometry showed that the apoptosis rate of combination group was significantly higher than the other two groups $(60.2 \% \pm 3.4 \%$ vs $23.7 \% \pm 2.3 \%$ vs $25 \% \pm 2.6 \%, P<0.05$, Figure $5 \mathrm{~B})$. Lentivirus-mediated shRNA targeting $\mathrm{Mcl}-1$ revealed prominent chemosensitizing activity combination with VCR against SNK-6 cells.

\section{Discussion}

Mcl-1 expresses widely in human cells, it blocks the progression of apoptosis by binding and sequestering the proapoptotic proteins Bak and Bax $[21,22]$, which are capable of forming pores in the mitochondrial membrane, allowing the release of cytochrome into the cytoplasm. Numerous, diverse cell-types including cancer cells have been shown to be reliant on Mcl-1 for their survival and development.

Studies about malignant lymphoma also showed that Mcl-1 overexpression played a pivotal role in oncogenesis through inhibition of apoptosis. It was reported that $\mathrm{Mcl}-1$ gene expresses generally and the level of its expression is positively related to the degree of malignancy in B cell lymphoma. Furthermore, it is a critical anti-apoptotic factor in T lymphocytes [23], myeloblastic leukaemia and myeloma cells. Recent research in 11 nasal NK/T-cell lymphoma cases found that two distinct Mcl1 expression groups existed for nasal NK/T-cell lymphoma, and Mcl-1 expression levels of the nasal NK/T-cell lymphoma group were similar to that of DLBCL and higher than that of FL groups [24].

In our study, NK/T-cell lymphoma cell line SNK-6 expressed Mcl-1 gene relatively higher by RT-PCR assay. As mentioned above, NK/T-cell lymphoma is highly ag- 


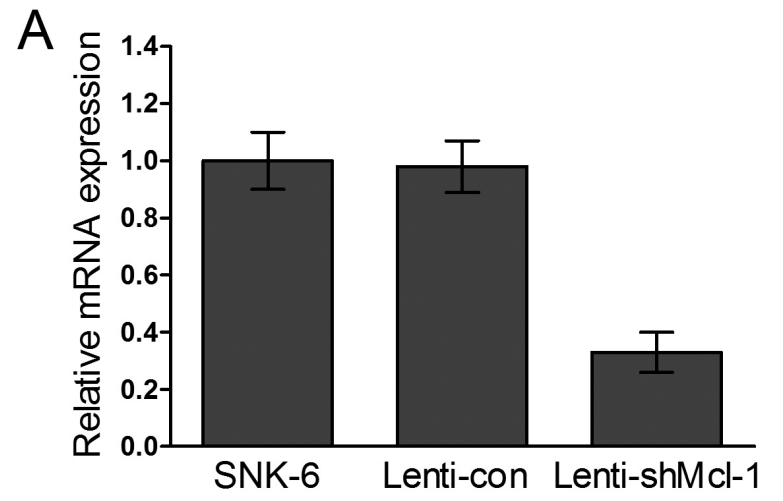

B

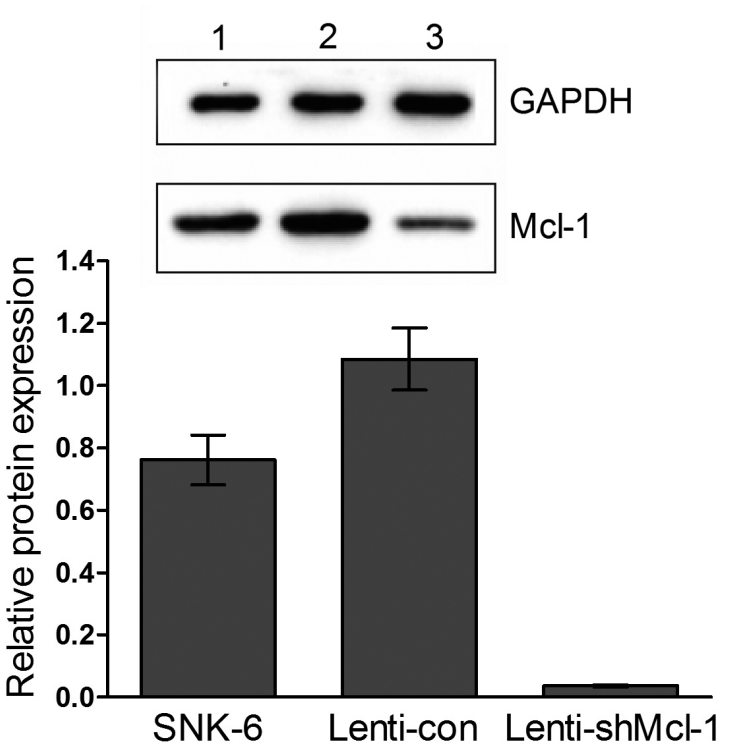

Figure 3. Expression of Mcl-1 after infection. (A) After $96 \mathrm{~h}$ infection mRNA levels of Mcl-1 in three groups were determined by quantitative real-time PCR $\left(2{ }^{-}{ }^{\mathrm{DCT}}\right)$. Results showed that mRNA levels were decreased significantly for lenti-shMcl-1 group. (B) Protein levels of Mcl-1 in three groups were determined by western blotting analysis (1: SNK-6, 2: lenticon, 3: lenti-shMcl-1), GAPDH was used as control. It showed that the protein levels were decreased by lenti-shMcl-1 remarkably.

gressive and the prognosis is poor. Thus we chose SNK-6 as our target cells. Recombinant lentiviruses which could efficiently infect SNK-6 cells were successfully constructed. MTT assay and flow cytometry were employed to detect the status of proliferation and apoptosis. The survival rate of Mcl-1 knock-down SNK-6 cells significantly decreased, no significant target regulation was observed for control groups. We could get that lentivirus-mediated shRNA targeting Mcl-1 has a prominent activity on growth inhibition against SNK-6 cells.

Microtubule-interfering agents are clinically important chemotherapeutic agent. Vinca alkaloids such as vincristine which was usually used in NK/T-cell lymphoma bind to the
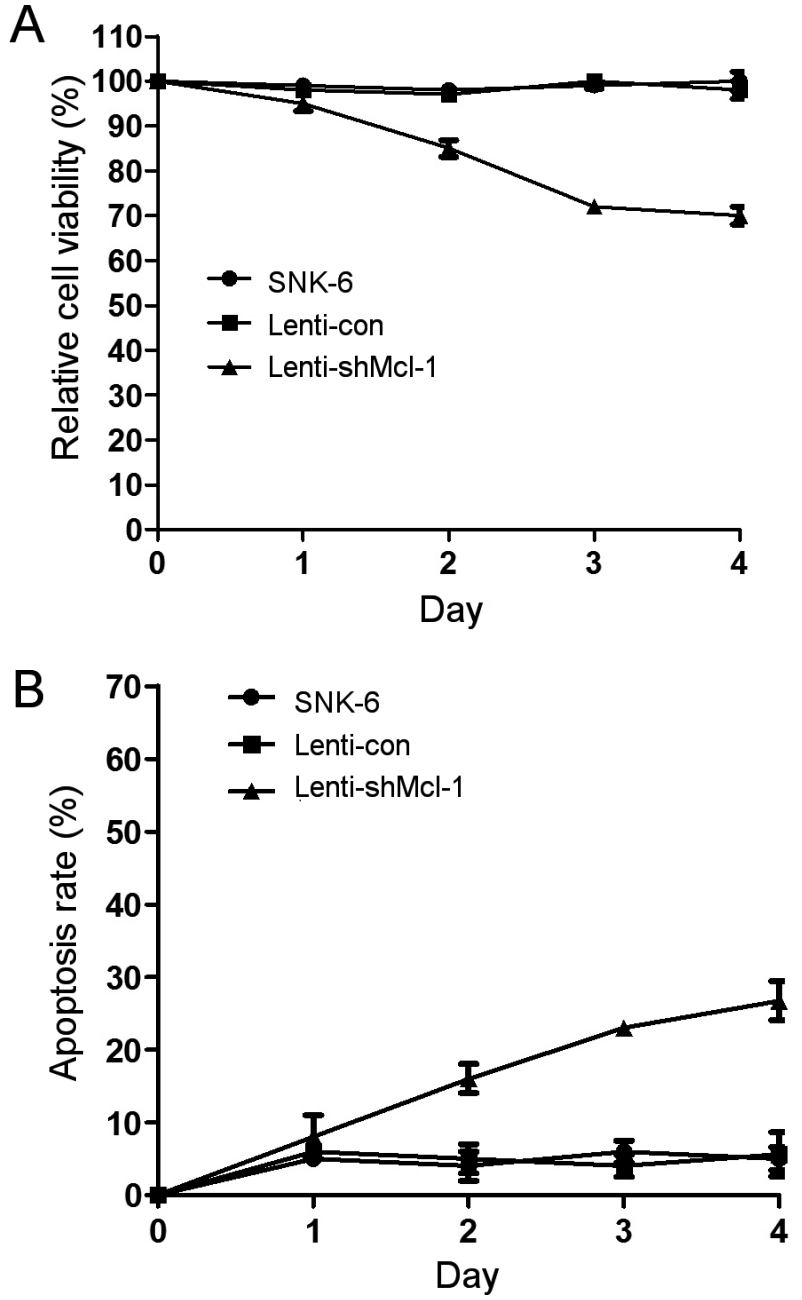

Figure 4. Effects of lenti-shMcl-1 on proliferation and apoptosis. (A) MTT assay was performed on SNK-6 cells for four successive days. The relative cell viability of three groups revealed that the survival rate of Mcl-1 knockdown cells was significantly lower than the control groups $(P<0.05)$. (B) Flow cytometry were employed to detect the status of apoptosis in three groups. It revealed that the apoptosis rate of Mcl-1 knockdown cells was significantly higher than the control groups $(P<0.05)$.

ends of microtubules, suppressing their dynamic instability and causing their depolymerization $[25,26]$. When used alone, vincristine is thought to induce cells to accumulate in mitosis prior to the induction of apoptosis, and these results in apoptosis occurring over a long period. Considering resistance to chemotherapy in most NK/T-cell lymphoma [27], in our study we investigated the effects of VCR combination with lenti-shMcl-1. Results showed that combination therapy induced significant apoptosis compared to single VCR therapy, down-regulation of Mcl-1 sensitized SNK-6 cells to low dose vincristine.

In conclusion, Mcl-1 was expressed widely in lymphoma cells. Specific downregulation of Mcl-1 using lentivirus-me- 

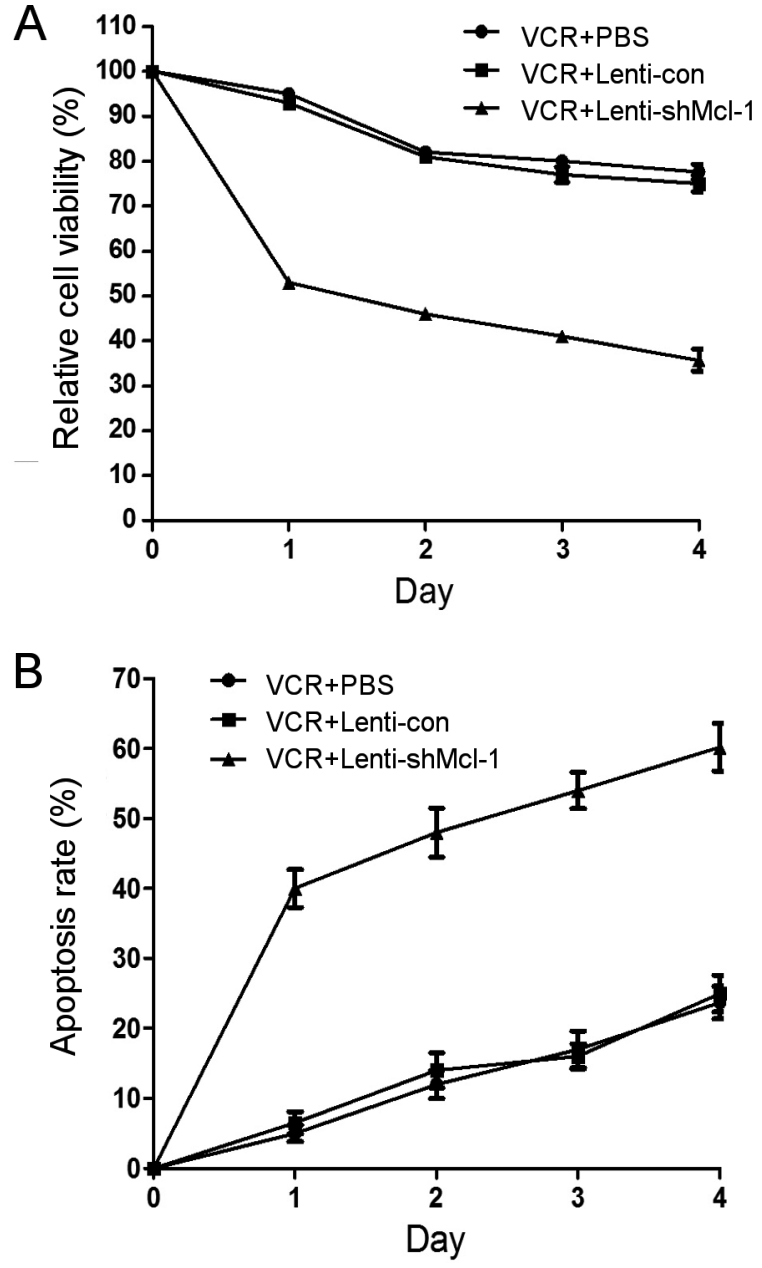

Figure 5. The effects of combination of lenti-shMcl-1 and vincristine (VCR). (A) The proliferation status of three groups (single VCR agent group and VCR combination with lenti-con or lenti-shMcl-1 group) was detected by MTT assay for four successive days. MTT assay suggested that the cell viability of SNK-6 cells was inhibited significantly by VCR combinated with lenti-shMcl-1 group after 4 days $(P<0.05)$. (B) Flow cytometry were employed to detect the status of apoptosis in three groups for four successive days. Results showed that the apoptosis rate of VCR combinated with lenti-shMcl-1 group was significantly higher than the two control groups $(P<0.05)$.

diated shRNA interference in SNK-6 cells lead to significant apoptosis induction and chemosensitization to vincristine. Thus, targeting Mcl-1 might qualify as a promising novel approach in treatment of NK/T-cell lymphoma. But further work is needed in vivo to prove the effects.

Acknowledgements: This study was supported by grants from the Key Science and Technology Research Project of Henan province (14B320033 and 201302011) and General Program of the National Natural Science Foundation of China (81470364 and 81570203).

\section{References}

[1] KWONG Y-L. The diagnosis and management of extranodal NK/T-cell lymphoma, nasal-type and aggressive NK-cell leukemia. J Clin Exp Hematop 2011; 51: 21-28. https://doi. org/10.3960/jslrt.51.21

[2] QUINTANILLA-MARTINEZ L, FRANKLIN JL, GUERRERO I, KRENACS L, NARESH KN et al. Histological and immunophenotypic profile of nasal NK/T cell lymphomas from Peru: high prevalence of p53 overexpression. Hum Pathol 1999; 30: 849-855. https://doi.org/10.1016/S00468177(99)90147-8

[3] KIM TM, HEO DS. Extranodal NK/T-cell lymphoma, nasal type: New staging system and treatment strategies. Cancer Sci 2009; 100: 2242-2248. https://doi.org/10.1111/j.13497006.2009.01319.x

[4] HE YF, WEI W, SUN ZM, JI CS, WANG G et al. Fatal lactic acidosis and hypoglycemia in a patient with relapsed natural killer/T-cell lymphoma. Adv Ther 2007; 24: 505-509. https:// doi.org/10.1007/BF02848772

[5] ISHIDA F, KWONG YL. Diagnosis and management of natural killer-cell malignancies. Expert Rev Hematol 2010; 3: 593-602. https://doi.org/10.1586/ehm.10.51

[6] TANI J1, FAUSTINE, SUFIAN JT. Updates on current advances in gene therapy. West Indian Med J 2011; 60: 188-194.

[7] YANG G, HUANG C, CAO J, HUANG KJ, JIANG T et al. Lentivirus-mediated shRNA interference targeting STAT3 inhibits human pancreatic cancer cell invasion. World J Gastroenterol 2009; 15: 3757-3766. https://doi.org/10.3748/ wjg.15.3757

[8] ZHANG X1, ZHAO L, HANG Z, GUO H, ZHANG M. Evaluation of HSV-1 and adenovirus vector-mediated infection, replication and cytotoxicity in lymphoma cell lines. Oncol Rep 2011; 26: 637-644.

[9] ZHANG X1, ZHAO L, CHEN C, YAN J, ZHOU C et al. The effect of lentivirus-mediated expression of tumor necrosis factor related apoptosis-inducing ligand and shRNA against Bcl-2 on the growth of lymphoma cells. Leuk Lymphoma 2012; 53: 710-717. https://doi.org/10.3109/10428194.2011.631158

[10] THOMAS LW, LAM C, EDWARDS SW. Mcl-1; the molecular regulation of protein function. FEBS Lett 2010; 584: 2981-2989. https://doi.org/10.1016/j.febslet.2010.05.061

[11] DANilov AV, DANilOVA OV, KLEIN AK, HUBER BT. Molecular pathogenesis of chronic lymphocytic leukemia. Curr Mol Med 2006; 6: 665-675. https://doi. org/10.2174/156652406778195008

[12] SIEGHART W, LOSERT D, STROMMER S, CEJKA D, SCHMID $\mathrm{K}$ et al. Mcl-1 overexpression in hepatocellular carcinoma: a potential target for antisense therapy. J Hepatol 2006; 44: 151-157. https://doi.org/10.1016/j. jhep.2005.09.010

[13] ZHOU P, LEVY NB, XIE H, QIAN L, LEE CY et al. MCL1 transgenic mice exhibit a high incidence of B-cell lymphoma manifested as a spectrum of histologic subtypes. Blood 2001; 97: 3902-3909. https://doi.org/10.1182/blood.V97.12.3902

[14] MOULDING DA, GILES RV, SPILLER DG, WHITE MR, TIDD DM et al. Apoptosis is rapidly triggered by antisense 
depletion of MCL-1 in differentiating U937 cells. Blood 2000; 96: $1756-1763$.

[15] AKGUL C. Mcl-1 is a potential therapeutic target in multiple types of cancer. Cell Mol Life Sci 2009; 66, 1326-1336. https:// doi.org/10.1007/s00018-008-8637-6

[16] SONG L, COPPOLA D, LIVINGSTON S, CRESS D, HAURA EB. Mcl-1 regulates survival and sensitivity to diverse apoptotic stimuli in human non-small cell lung cancer cells. Cancer Biol Ther 2005; 4: 267-276. https://doi.org/10.4161/ cbt.4.3.1496

[17] FU JR, LIU WL, ZHOU JF, SUN HY, ZHENG M et al. [The effects of Mcl-1 gene on ATRA-resistant HL-60 cell]. Zhonghua Xue Ye Xue Za Zhi 2005; 26: 352-354.

[18] THALLINGER C, WOLSCHEK MF, MAIERHOFER H, SKVARA H, PEHAMBERGER $\mathrm{H}$ et al. Mcl-1 is a novel therapeutic target for human sarcoma: synergistic inhibition of human sarcoma xenotransplants by a combination of mcl-1 antisense oligonucleotides with low-dose cyclophosphamide. Clin Cancer Res 2004; 10: 4185-4191. https://doi. org/10.1158/1078-0432.CCR-03-0774

[19] ALVES NL, DERKS IA, BERK E, SPIJKER R, VAN LIER RA et al. The Noxa/Mcl-1 axis regulates susceptibility to apoptosis under glucose limitation in dividing T cells. Immunity 2006; 24: 703-716. https://doi.org/10.1016/j.immuni.2006.03.018

[20] SASTRY L, JOHNSON T, HOBSON MJ, SMUCKER B, CORNETTA K. Titering lentiviral vectors: comparison of DNA, RNA and marker expression methods. Gene Ther 2002; 9: 1155-62. https://doi.org/10.1038/sj.gt.3301731

[21] OLTVAI ZN, MILLIMAN CL, KORSMEYER SJ. Bcl-2 heterodimerizes in vivo with a conserved homolog, Bax, that accelerates programed cell death. Cell 1993; 74: 609-619. https://doi.org/10.1016/0092-8674(93)90509-O

[22] STROBEL T, SWANSON L, KORSMEYER S, CANNISTRA SA. BAX enhances paclitaxel-induced apoptosis through a p53-independent pathway. Proc Natl Acad Sci U S A 1996; 93: 14094-14099. https://doi.org/10.1073/pnas.93.24.14094

[23] Dunkle A, Dzhagalov I, HE YW. Cytokine-dependent and cytokine-independent roles for Mcl-1: genetic evidence for multiple mechanisms by which Mcl-1 promotes survival in primary T lymphocytes. Cell Death Dis 2011; 2: e214. https:// doi.org/10.1038/cddis.2011.95

[24] YASUDA H, SUGIMOTO K, IMAI H, ISOBE Y, SASAKI M et al. Expression levels of apoptosis-related proteins and Ki67 in nasal NK/T-cell lymphoma. Eur J Haematol 2009; 82: 39-45. https://doi.org/10.1111/j.1600-0609.2008.01152.x

[25] FAN M, GOODWIN M, VU T, BRANTLEY-FINLEY C, GAARDE WA et al. Vinblastine-induced phosphorylation of $\mathrm{Bcl}-2$ and $\mathrm{Bcl}-\mathrm{XL}$ is mediated by JNK and occurs in parallel with inactivation of the Raf-1/MEK/ERK cascade. J Biol Chem 2000; 275: 29980-29985. https://doi.org/10.1074/jbc. M003776200

[26] YAMAMOTO K, ICHIJO H, KORSMEYER SJ. BCL-2 is phosphorylated and inactivated by an ASK1/Jun N-terminal protein kinase pathway normally activated at G2/M. Mol Cell Biol 1999; 19: 8469-8478. https://doi.org/10.1128/ MCB.19.12.8469

[27] YECIES D, CARLSON NE, DENG J, LETAI A. Acquired resistance to ABT-737 in lymphoma cells that up-regulate MCL-1 and BFL-1. Blood 2010; 115: 3304-3313. https://doi. org/10.1182/blood-2009-07-233304 